\title{
Early life metal exposure dysregulates cellular bioenergetics in children with regressive autism spectrum disorder
}

\author{
Richard E. Frye $\mathbb{B}^{1,2}$, Janet Cakir ${ }^{3}$, Shannon Rose $\mathbb{C}^{4,5}$, Leanna Delhey $\mathbb{B}^{4,5}$, Sirish C. Bennuri, ${ }^{4,5}$, Marie Tippett ${ }^{4,5}$, \\ Raymond F. Palmer, Christine Austin ${ }^{7}$, Paul Curtin ${ }^{7}$ and Manish Arora ${ }^{7}$
}

\begin{abstract}
Neurodevelopmental regression (NDR) is a subtype of autism spectrum disorder (ASD) that manifests as loss of previously acquired developmental milestones. Early life dysregulation of nutritional metals and/or exposure to toxic metals have been associated with ASD, but the underlying biological mechanisms by which metals influence neurodevelopment remain unclear. We hypothesize that metals influences neurodevelopment through dysregulation of bioenergetics. Prenatal and early postnatal metal exposures were measured using validated tooth-matrix biomarkers in 27 ASD cases (13 with NDR) and 7 typically-developing (TD) controls. Mitochondrial respiration and glycolysis were measured in peripheral blood mononuclear cells using the Seahorse XF96. Children with ASD demonstrated lower prenatal and postnatal Copper (Cu) and prenatal Nickel concentrations and Copper-to-Zinc (Cu/Zn) ratio as compared with TD children. Children with ASD and NDR showed greater metal-related disruption of cellular bioenergetics than children with ASD without NDR. For children with ASD and NDR mitochondrial respiration decreased as prenatal Manganese concentration increased and increased as prenatal Zinc concentration increased; glycolysis decreased with increased exposure to prenatal Manganese and Lead and postnatal Manganese. For children with ASD without a history of NDR, glycolysis increased with increased postnatal exposure to Tin. Language and communication scores in children with ASD were positively related to prenatal $\mathrm{Cu}$ exposure and Cu/Zn ratio. This study suggests that prenatal nutritional metals may be important for neurodevelopment in children with ASD, and that exposure to toxic metals and differences in nutritional metal exposures is associated with dysregulation of cellular bioenergetics, particularly in the NDR subtype of ASD.
\end{abstract}

\section{Introduction}

Autism spectrum disorder (ASD) is a behaviorally defined disorder, which is associated with significant pathophysiol$\mathrm{ogy}^{1}$. Some studies estimate ASD to affect 1 in 45 individuals in the United States ${ }^{2}$. Although ASD research has focused on genetics ${ }^{1}$, recent estimates suggest that inherited single gene and chromosomal defects only account for a minority of ASD cases $^{3}$ and that ASD most likely arises from a complicated interaction between genetic predisposition and

Correspondence: Richard E. Frye (rfrye@phoenixchildrens.com)

'Barrow Neurological Institute at Phoenix Children's Hospital, Phoenix, AZ, USA

${ }^{2}$ University of Arizona College of Medicine - Phoenix, Phoenix, AZ, USA

Full list of author information is available at the end of the article the environment ${ }^{4,5}$. Although numerous environmental agents have been epidemiologically associated with ASD, the mechanism by which such agents cause neurodevelopmental disorders is understudied and unclear ${ }^{6}$.

Commonly studied environmental toxicants include gestation and early-life exposure to fine air particulate matter (e.g., $\mathrm{PM}_{2.5}$ ), which is linked to an increase in ASD risk $^{7-9}$. Important nutrients linked to ASD include folate, which increases the risk for ASD when not provided in sufficient amounts during gestation ${ }^{10}$. Recent research has demonstrated that exposure to metals, which can be nutritional and/or toxic during gestation and early life, can increase the risk of developing $\mathrm{ASD}^{11,12}$. Lead $(\mathrm{Pb})$ 
exposure is associated with neurodevelopmental disorders including behavioral problems, learning deficits, intellectual disability, and $\mathrm{ASD}^{13}$. Other metals such as Zinc (Zn), Copper $(\mathrm{Cu})$, and Manganese $(\mathrm{Mn})$ are essential for metabolic systems and neurodevelopment but can be toxic in excessive amounts ${ }^{14}$. A recent major advancement that has helped with the study of exposure to metals prenatally and during early life is the ability to directly measure exposure with a week-by-week temporal resolution during critical developmental periods by examining metal deposits in deciduous teeth ${ }^{15}$. Recent studies using deciduous teeth have confirmed that prenatal disruption in these metals are linked to an increase in the risk of developing $\mathrm{ASD}^{11,12}$.

Although toxic and nutritional metal exposure has been associated with an increased risk of neurodevelopmental and neuropsychiatric disorders, the exact pathophysiological mechanisms by which metal exposure contributes to these abnormalities are mostly based on animal models and not well understood in humans. One important mechanism by which environmental influences, especially those linked to ASD, can affect biological systems is through mitochondrial metabolism ${ }^{6,16}$. This is compelling since up to $30-50 \%$ of individuals with ASD demonstrate biomarkers of abnormal mitochondrial function ${ }^{16,17}$, and up to $80 \%$ of individuals with ASD show abnormal electron transport chain (ETC) activity in lymphocytes ${ }^{18}$, granulocytes $^{19}$, and post-mortem brain ${ }^{20}$. Furthermore, the great majority of cases of individuals with ASD and mitochondrial disease do not have identified genetic mutations, suggesting that alternations in mitochondrial function may be driven from nongenetic influences such as the environment ${ }^{16}$. Interestingly, the same environmental agents that have been linked to ASD can also induce mitochondrial metabolism abnormalities ${ }^{21}$. Furthermore, mitochondria adapt to changes in the microenvironment through a process called mitoplasticity, which has the potential to result in long-term changes to mitochondrial function ${ }^{22}$.

In this study, we directly measure both toxic and nutrient metals deposited into each participant's deciduous teeth during gestation and early in infancy (first 9 months of age) to determine if such metals have a long-term association with changes in bioenergetics in children with ASD. About one-third of children with ASD fall into the neurodevelopmental regression (NDR) subtype. Children with NDR demonstrate normal development in infancy but then lose social and language skills, usually between the first and second years of life. Because NDR is associated with mitochondrial dysfunction in children with ${ }^{16,23}$ and without $^{24}$ ASD, we pay special attention to children with ASD diagnosed with NDR.

As the mitochondria is particularly sensitive to environmental factors such as nutrient and toxic metal exposure, and since mitochondrial dysfunction is linked to NDR, it is hypothesized that prenatal and early postnatal exposure to metals will result in long-term changes in mitochondrial function that is sustained into childhood, particularly in those children with ASD and NDR. In this study, we demonstrate that metal exposure during gestation is related to physiological changes in bioenergetics that persist into childhood for children with ASD, primarily in those with a history of NDR. These results suggest that early life environmental exposures are linked to long-term physiological abnormalities in a subset of children with ASD.

\section{Methods \\ Participants}

Protocols registered in clinicaltrials.gov as NCT020002 84 and NCT02003170 and approved by the Institutional Review Board at the University of Arkansas for Medical Sciences (Little Rock, AR) were used in this study. Exclusion criteria were (i) chronic treatment with medications that would detrimentally affect mitochondrial function such as antipsychotic medications; (ii) vitamin or mineral supplementation exceeding the recommended daily allowance, and (iii) prematurity.

Inclusion criteria included: (a) a diagnosis of ASD and (b) $<18$ years of age. The ASD diagnosis was defined by one of the following: (i) a gold-standard diagnostic instrument: the Autism Diagnostic Observation Schedule or Autism Diagnostic Interview-Revised; (ii) the state of Arkansas diagnostic standard, defined as agreement of a physician, psychologist, and speech therapist with experience in diagnosing ASD; and/or (iii) Diagnostic Statistical Manual diagnosis by a physician along with standardized validated questionnaires and diagnosis confirmation by the first author (REF). We have previously validated that criteria (ii) and (iii) captures an accurate diagnosis of ASD by showing that participants defined using criteria (ii) or (iii) are well within the diagnostic criteria for ASD using the Autism Diagnostic Interview-Revised ${ }^{25}$.

Parents of participants provided written informed consent. Children underwent a fasting blood draw in the morning. Control individuals did not have any neurological disorders or developmental delays and none of the individuals included in this study were diagnosed with mitochondrial disease. Patients were recruited from a nationally recognized multispecialty ASD clinic. Patients lived in one of eight states (AL, AR, FL, GA, IL, KY, MO, and TX) or Canada. Supplementary Table S1 outlines the participant characteristics. Our recent publications outline the methodology for rating participant characteristics of this participant cohort ${ }^{25-28}$. The numerous medical conditions were summarized into categories. Feeding problems, diarrhea, constipation, stomach and/ or abdominal pain, vomiting, and gastroesophageal reflux disease were considered gastrointestinal disorders. 
Asthma, allergies, allergic skin conditions, breathing problems, and other lung problems were considered allergic disorders. Depressive, anxiety, bipolar, obsessive compulsive, and attention deficit with or without hyperactivity disorders were considered psychiatric disorders. Recurrent sinusitis, ear infections, throat infections, and other immune problems were considered immune disorders. Headaches, hearing problems, microcephaly, vision problems, macrocephaly, strabismus, seizures, epilepsy, opthalmoplegia, tics, cerebral palsy, and muscle weakness were considered neurological disorders. Cardiovascular disorders are considered syncope, heart failure, and congenital heart disease. Growth disorders are considered failure to thrive, growth hormone deficiency, short and tall stature, overweight, and obesity. Endocrine disorders are considered thyroid or other endocrine problems.

The NDR history was obtained using the Developmental and Neurobehavioral Regression (DANR) questionnaire, which has been developed as part of our ASD research program. The DANR records detail information about NDR including specific questions on premorbid functioning before the regression, duration of the regression, specific skills lost and when the skills were regained, whether there was a single or multiple regressions and any known trigger such as illness, fever, or seizure. The two ASD groups were similar in characteristics except for gastrointestinal medications, which were used in a greater percentage of children with ASD and NDR as compared with those with ASD without NDR.

Contemporaneous controls were slightly younger and had more females than the children with ASD. The control children were generally healthy children without significant chronic health conditions although one had a history of strabismus, two had a history of headache, one had anxiety, and several had seasonal allergies. Most (5 of 7) of these individuals underwent the Vineland Adaptive Behavior Scale 2nd Edition to document their normal development. In addition, the controls were screened with the social communication questionnaire to document the lack of ASD symptoms with a total score $<12$.

Metal exposure was measured from a total of 34 children, including 7 who were typically developing (TD) [2 siblings of children with ASD and 5 unrelated to the children with ASD]. Neither sex nor age were significant covariates in any analysis. Mitochondrial respiration and glycolysis were measured in 27 children who were diagnosed with ASD without known mitochondrial disease. Some children provided repeated blood samples for analysis: 17 provided one sample, 9 provided two samples and 1 provided three or more samples. Participants were only required to provide one blood sample but could provide up to five repeated samples.

\section{Neurodevelopmental and behavioral measurements}

Common validated measures were used to assess neurodevelopment and behavior in children with $\mathrm{ASD}^{25,29}$. Core language was assessed by the most appropriate instrument given the participants age, either the Clinical Evaluation of Language Fundamentals (CELF)-preschool-2, CELF-4, or the Preschool Language Scale-5 (PLS-5). The standardized summary score of each instrument (mean 100, standard deviation 15) was used as the measure of core language similar to our previous study ${ }^{25}$. The Vineland Adaptive Behavior Scale (VABS) 2nd edition Survey Interview Form was used as a measure of neurodevelopment. Parents completed the Aberrant Behavior Checklist (ABC) and the Social Responsiveness Scale (SRS) as measures of behavior and ASD symptoms. For language and VABS, higher scores represent higher functioning and for the SRS and $A B C$ lower scores represent less impairment.

\section{Measurements of prenatal metal exposure}

Elemental bio-imaging using laser ablation-inductively coupled plasma mass-spectrometry (LA-ICP-MS) measured concentrations of $\mathrm{Pb}$ (lead), $\mathrm{Ni}$ (nickel), Cr (chromium), $\mathrm{Mg}$ (magnesium), $\mathrm{Mn}$ (manganese), $\mathrm{Cu}$ (copper), $\mathrm{Zn}$ (zinc), Sr (strontium), Sn (tin), and $\mathrm{Ba}$ (barium) in deciduous teeth with a trimester-by-trimester resolution $^{30-33}$. We also calculated the $\mathrm{Cu}$-to- $\mathrm{Zn}$ ratio. Many studies in ASD have used the $\mathrm{Zn}$-to-Cu ratio but we found in tooth data that the $\mathrm{Zn}$-to- $\mathrm{Cu}$ ratio had very wide variability and a non-normal distribution. Thus, we used the $\mathrm{Cu}$-to- $\mathrm{Zn}$ ratio which demonstrates a more normal distribution.

The LA-ICP-MS system includes an Agilent 8800 ICPMS and a New Wave Nd:YAG $193 \mathrm{~nm}$ laser ablation system with a detection limit of $0.05 \mu \mathrm{g} / \mathrm{g}$ for the metals studied (Fig. 1). All samples analyzed include calibration standards and quality control (QC) samples. Certified reference materials (e.g., NIST 2974 freeze-dried tissue for persistent pollutants and NIST 3673 non-smokers' urine for phthalates, phenols, and tobacco metabolites) are used for $\mathrm{QC}$ and method validation. Isotopically labeled internal standards monitor analyte recovery during sample preparation and are used to correct for matrix-effects during analysis. Either gas chromatography (GC)-MS/MS or liquid chromatography (LC)-MS/MS determined analytes, depending on sensitivity and lowest detection limit achievable. Traditionally, polar analytes are determined by LC, and non-polar and volatiles by GC. Tooth metal biomarkers, particularly the method used in this study, has been utilized in previous studies of prenatal metal exposure $^{11,12}$. Higher values indicate higher metal concentrations in the tooth at a particular temporal window.

Tooth metal biomarkers have been validated using several methods. First, abrupt changes in $\mathrm{Mn}$ and $\mathrm{Zn}$ concentrations occur at birth; tooth-matrix biomarkers 

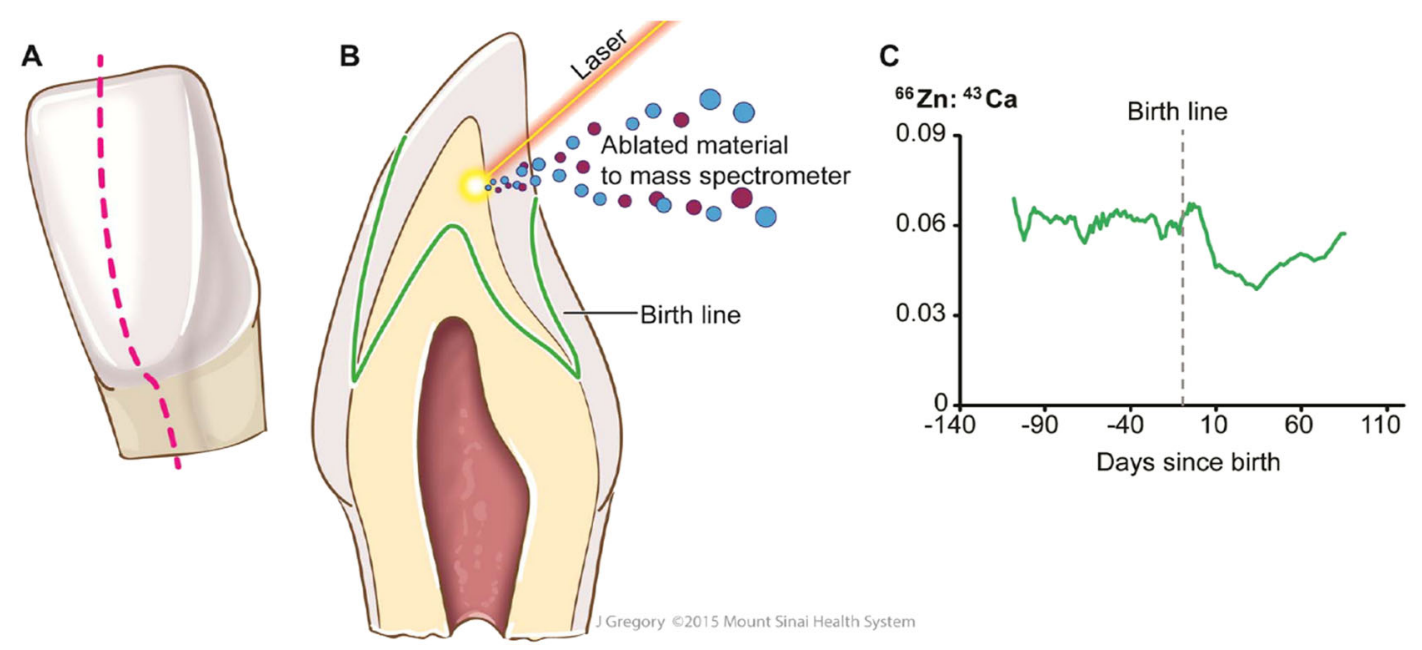

Fig. 1 Overview of tooth-matrix biomarkers applied in this study, a Plane in which teeth were sectioned. $\mathbf{b}$ Laser ablation-inductively coupled plasma mass spectrometry analysis. c Output data where developmental timing is assigned to elemental profiles in each sample.

have demonstrated that this abrupt change in $\mathrm{Mn}$ and $\mathrm{Zn}$ concentrations in deciduous teeth is congruent with the neonatal line, a histological feature formed in enamel and dentine ${ }^{11,34,35}$. Second, monozygotic twin studies have verified the correspondence between fetal exposure and metal deposits in deciduous teeth ${ }^{11}$. Third, metal deposition in teeth has been compared with other environmental matrices in prospective pregnancy cohorts ${ }^{15,32,34-36}$. Fourth, blood lead levels measured from the mother prenatally and children postnatally correspond to deciduous tooth measurements ${ }^{15}$. Finally, animal studies with controlled exposures have been used to validate the method ${ }^{35,37}$.

\section{Blood collection and processing}

Up to $20 \mathrm{ml}$ of blood was collected into an ethylenediaminetetraacetic acid-Vacutainer tube, chilled on ice and centrifuged at $1500 \mathrm{~g}$ for $10 \mathrm{~min}$ at $4{ }^{\circ} \mathrm{C}$ to separate plasma within $15 \mathrm{~min}$ of collection. Plasma was removed and stored at $-80^{\circ} \mathrm{C}$ for later analysis. Plasma was replaced with room temperature wash buffer containing $\mathrm{Ca}^{+2} / \mathrm{Mg}^{+2}$-free PBS with $0.1 \% \mathrm{BSA}$ and $2 \mathrm{mM}$ ethylenediaminetetraacetic acid. Diluted blood was then layered on top of Histopaque-1077 (Sigma Aldrich, St. Louis, MO, USA) and centrifuged at $400 \mathrm{~g}$ for $30 \mathrm{~min}$ at room temperature. Peripheral blood mononuclear cells (PBMCs) were washed twice with wash buffer and counted using a hemocytometer.

\section{Bioenergetic assay}

Bioenergetic data was obtained from PBMCs using a stateof-the-art Seahorse 96XF Analyzer (Seahorse Bioscience, Inc., North Billerica, MA) that measures oxygen consumption rate in real-time in a 96-well plate. This analyzer evaluates a wide range of intact living cell types ${ }^{38,39}$ and can measure ETC, glycolytic, and fatty-acid oxidation function ${ }^{40}$.
The assay measures several key respiratory parameters: (a) Adenosine triphosphate-Linked Respiration, (b) Proton leak Respiration, (c) Maximal Respiratory Capacity, a parameter that is sensitive to deficits in mitochondrial biogenesis, mtDNA damage and/or inhibition of ETC function, and (d) Reserve Capacity, a parameter which determines the threshold at which bioenergetic dysfunction occurs. Measure of glycolysis are also measured. The extracellular acidification rate, a measure of lactate production, is equated to the glycolytic rate (i.e., glycolysis) and measured simultaneously with oxygen consumption rate in the Seahorse assay before the injection of oligomycin.

PBMCs were placed in assay media (unbuffered RPMI supplemented with $1 \mathrm{mM}$ pyruvate, $2 \mathrm{mM}$ glutamate, and $25 \mathrm{mM}$ glucose) and plated at $\sim 4 \times 10^{5}$ cells per well of a poly-D-lysine coated XF96 well plate. For each experimental condition, four samples were measured simultaneously to improve assay reliability. Runs with clear measurement probe failure or reagent injection failure were eliminated. Overall, there was a total of 120 bioenergetic measurements used in the analysis.

\section{Statistical analysis}

Analyses were performed using SAS 9.4 (SAS Institute Inc., Cary, NC) and PAWS Statistics 18 (SPSS Inc, Quarry Bay, HK). Graphs were produced using Excel version 16.0 (Microsoft Corp, Redmond, WA). In general, several personnel specializing in specific parts of data collection and analysis were used to maintain blinding to the outcome measures when possible. Specifically the blood was collected and relevant clinical information was obtained by different people (L.D., M.T.) from the laboratory personnel who performed the mitochondrial respiratory measurement (S.R., S.C.B.) and the laboratory that performed the tooth 
analysis (M.A.). Data from all sources was compiled by nonlaboratory staff (L.D., M.T.) and statistical analysis was performed by individuals not directly involved in sample collection or laboratory analysis of the samples (J.C., R.E.F.).

A linear mixed-model was used to account for both within-subject variation from repeated mitochondrial measurements on the same individual (replicates) and the between-subject variation from variables such as NDR and metal concentration. Variances between groups were similar and data was normally distributed. All interactions were considered in the models and the final models were simplified to eliminate any non-significant higher order interactions. Because of the multiple metals analyzed the overall $p$ was set to $\leq 0.01$ for all analyses. To verify this approach, we calculated the False Discovery Rate for each set of statistical tests for each respiratory parameter on all metals. A False Discovery Rate of $5 \%$ is consistent with setting an $\alpha \leq 0.01$ significance level $^{41}$.

Since multiple mitochondrial respiratory measurements were investigated, the metal investigated needed to demonstrate significance for at least three of the four respiratory measurements. This is a conservative approach to decreasing false positives. Since only one measure of glycolysis was investigated, this increase stringent criteria of having multiple respiratory parameters positive could not be set for the glycolysis analysis.

In the case of an interaction between NDR with metal concentration, the $p$ value for the follow-up correlation between the respiratory parameter and the metal concentration for the group (NDR or not NDR) was required to be $\leq 0.01$. To confirm and complement the mixedmodel analysis, bivariate Pearson correlation coefficient $r$ along with the $p$ value for the $r$ are presented in the scatterplot graphs. The $r$ values can be used as a representation of effect size since $r$ values are already standardized measures of the strength of the relationship ${ }^{42}$. Accordingly, $r$ of $0.1-0.3$ is recognized as a small effect, $r$ of 0.3-0.5 is recognized as a medium effect and $r$ of 0.50 or above is recognized as a large effect.

It is important to realize that the small sample size of this study limits the power of the statistical analyses. Thus, we cannot rule out relationships that are not found to be statistically significant. However, the purpose of this study is to determine if there are relationships that do exist given the conservative false positive rate (i.e., $\leq 1 \%$ ).

As an exploratory analysis we have examined the relationship between neurodevelopmental and behavioral measures, and metals found to be important in ASD and/ or mitochondrial function using linear regression including NDR as a moderating variable. For this exploratory analysis, alpha was set at 5\%. Follow-up bivariate Pearson correlation were also computed to verify the analysis with relationships requiring an alpha of $5 \%$.

\section{Results}

We compared the concentration of metals in deciduous teeth between children with ASD and TD control children as well as between children with ASD with and without a history of NDR (See Supplementary Table S2). There were no differences in tooth metal concentrations in the prenatal or postnatal period between those with and without NDR. However, compared with TD children, ASD children demonstrated significantly lower $\mathrm{Ni}, \mathrm{Cu}$, and $\mathrm{Cu}$-to- $\mathrm{Zn}$ ratio during the prenatal period and lower $\mathrm{Cu}$ during the early postnatal period. $\mathrm{Zn}$ and $\mathrm{Sn}$ were also lower in ASD children during the prenatal period, although the difference did not reach our stringent $(p \leq$ $0.01)$ criteria (See Supplementary Material for Statistics).

\section{Mitochondrial respiration and prenatal metal exposure}

Two metals, $\mathrm{Zn}$ and $\mathrm{Mn}$, demonstrated significant relationships for three or more respiratory parameters with these relationships dependent on whether the child had a history of NDR (See Supplementary Table S3). Interestingly, when controlling for $\mathrm{Zn}$ and $\mathrm{Mn}$ concentration, all measures of mitochondrial respiration were found to be significantly higher in the children with NDR as compare with those without NDR, further demonstrating that differences in these metals significantly affect mitochondrial respiration (See Supplementary Tables S3 and S4). As seen in Fig. 2a-d, greater Zn deposited in deciduous teeth prenatally was associated with higher mitochondrial respiration for those individuals with a history of NDR but there was no significant relationship between $\mathrm{Zn}$ and mitochondrial respiration for those without a NDR history. As seen in Fig. 2e-h, greater Mn deposited in deciduous teeth prenatally was associated with lower mitochondrial respiration for those individuals with a history of NDR but there was no significant relationship between $\mathrm{Mn}$ and mitochondrial respiration for those without a history of NDR.

\section{Mitochondrial respiration and postnatal metal exposure}

Two metals $(\mathrm{Ni}, \mathrm{Sr})$ demonstrated significant $(p<0.01)$ relationships for three or more respiratory parameters, with these relationships dependent on whether the child had a history of NDR. However, the scatterplots demonstrated that these relationships were driven by one child with particularly high concentrations of both metals. When this participant was removed, the analysis was no longer significant according to our criteria although a trend remained (data not shown).

\section{Glycolysis and prenatal metal exposure}

Glycolytic rate was associated with prenatal deciduous tooth concentrations of $\mathrm{Mn}$ and $\mathrm{Pb}$. The effect of $\mathrm{Mn}$ $[F(1,113)=6.68, p=0.01]$ and $\mathrm{Pb}[F(1113)=6.37, p=$ $0.01]$ on glycolytic rate was dependent on the participant's 

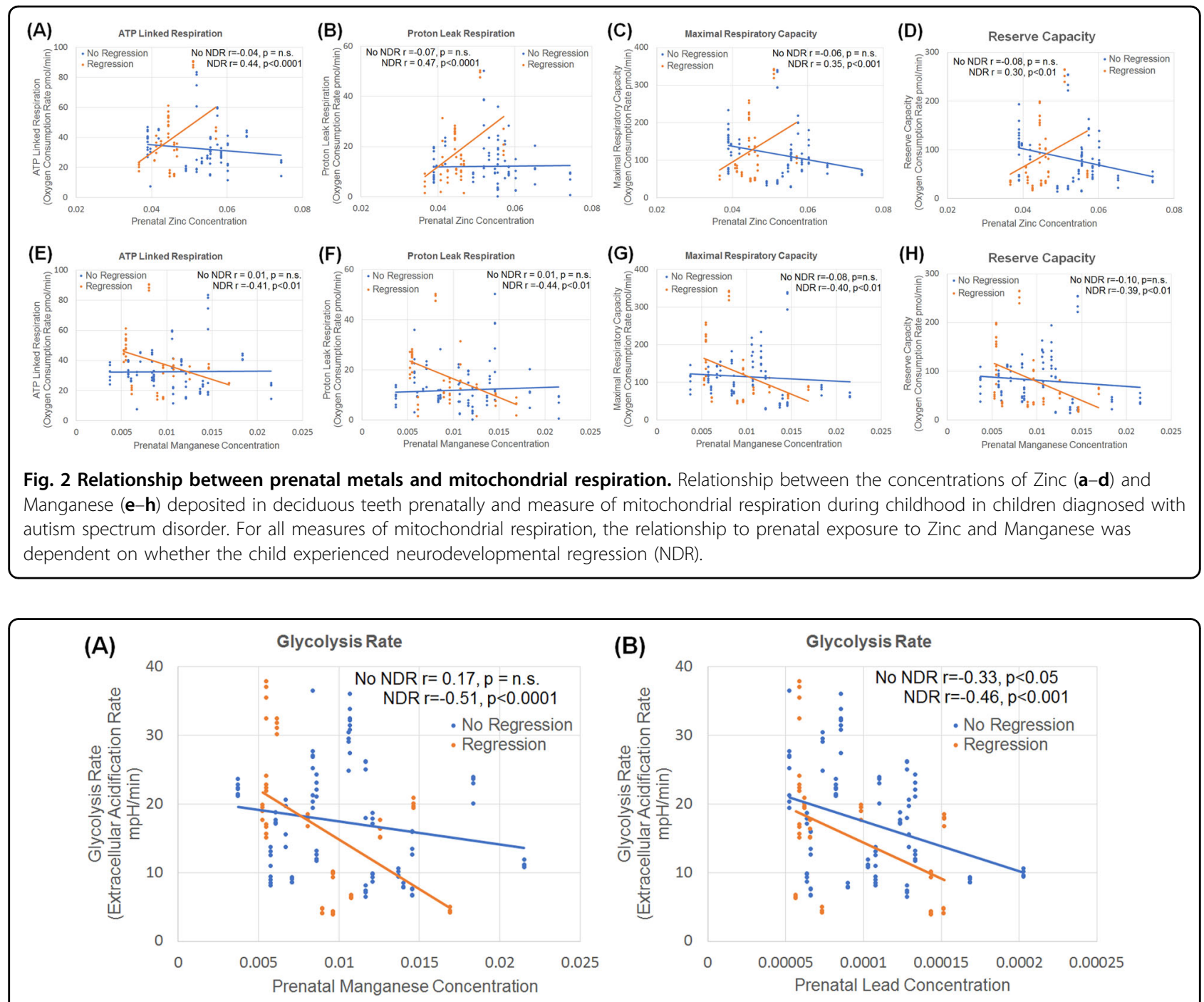

Fig. 3 Relationship between prenatal metals and glycolysis. Relationship between Glycolytic rate and prenatal concentrations of Manganese and Lead for children with autism spectrum disorder. Prenatal concentration of (a) Manganese and (b) Lead are related to glycolysis but the relationship between metal concentrations and glycolytic rate was dependent on whether the child experienced neurodevelopmental regression (NDR).

history of NDR. The glycolytic rate decreased with an increasing concentration of Mn (Fig. 3a) and Pb (Fig. 3b) for those with a history of NDR. For those without a history of NDR, there was no significant relationship between glycolytic rate and deciduous Mn concentration while the relationship between $\mathrm{Pb}$ and glycolytic rate was present but weaker than those with a history of NDR.

\section{Glycolysis and postnatal metal exposure}

Glycolytic rate was associated with early postnatal deciduous tooth concentrations of $\mathrm{Mn}$ and $\mathrm{Sn}$. The effect of $\operatorname{Mn}[F(1113)=9.96, p<0.01]$ and $\operatorname{Sn}[F(1113)=10.68$, $p<0.01]$ on glycolytic rate was dependent on the participant's history of NDR. The glycolytic rate decreased with an increasing concentration of Mn (Fig. 4a) for those with a history of NDR but was not associated with the glycolytic rate for those without a history of NDR. In contrast, the glycolytic rate increased with an increasing concentration of Sn (Fig. 4b) for those without a history of NDR but was not associated with the glycolytic rate for those with a history of NDR.

\section{Behavioral correlates of metals}

Core language and VABS Communication subscale scores increased as prenatal $\mathrm{Cu}$ concentration $[F(123)=$ 4.65, $p<0.05$ and $F(123)=4.91, p<0.05]$ and $\mathrm{Cu}$-to- $\mathrm{Zn}$ ratio $[F(123)=6.31, p<0.05$ and $F(123)=5.76, p<0.05]$ increased (Fig. 5).

\section{Discussion}

Exposure to nutrient (e.g., $\mathrm{Zn}$ ) and toxic (e.g., $\mathrm{Pb}$ ) metals are known to be associated with ASD yet the biological 

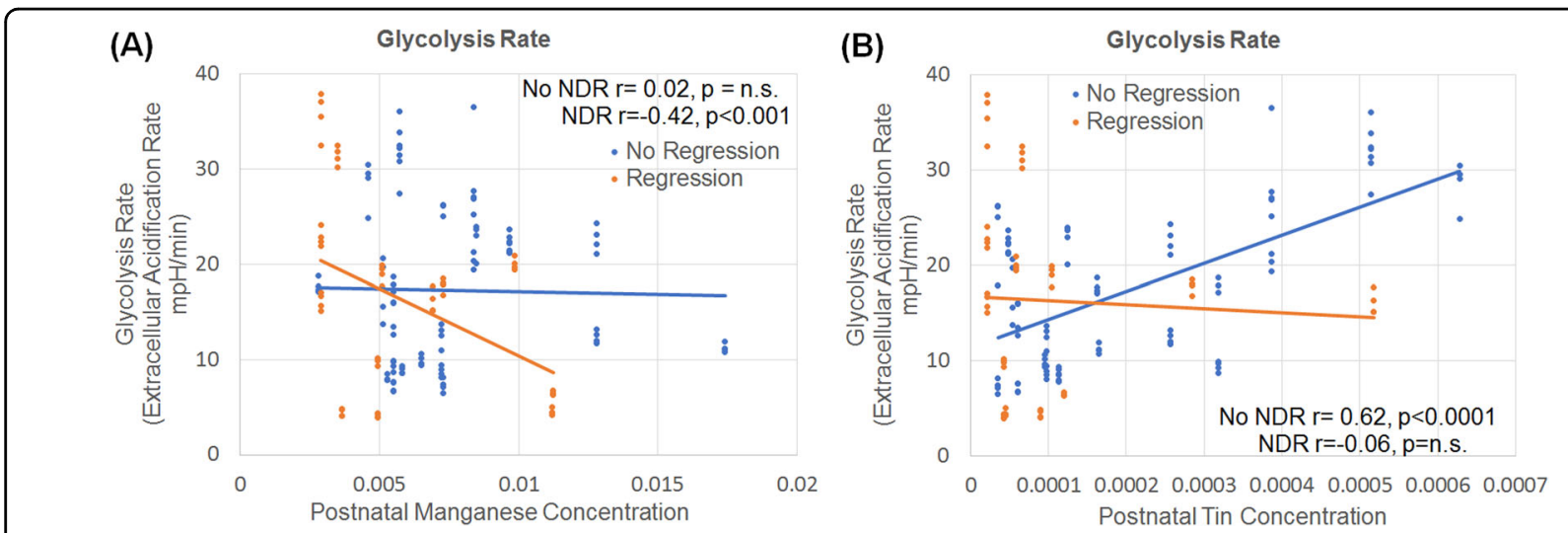

Fig. 4 Relationship between postnatal metals and glycolysis. Relationship between Glycolytic rate and early postnatal (first 9 months of life) concentrations of (a) Manganese and (b) Tin for children with autism spectrum disorder. The relationship between metal concentrations and glycolytic rate was dependent on whether the child experienced neurodevelopmental regression (NDR).

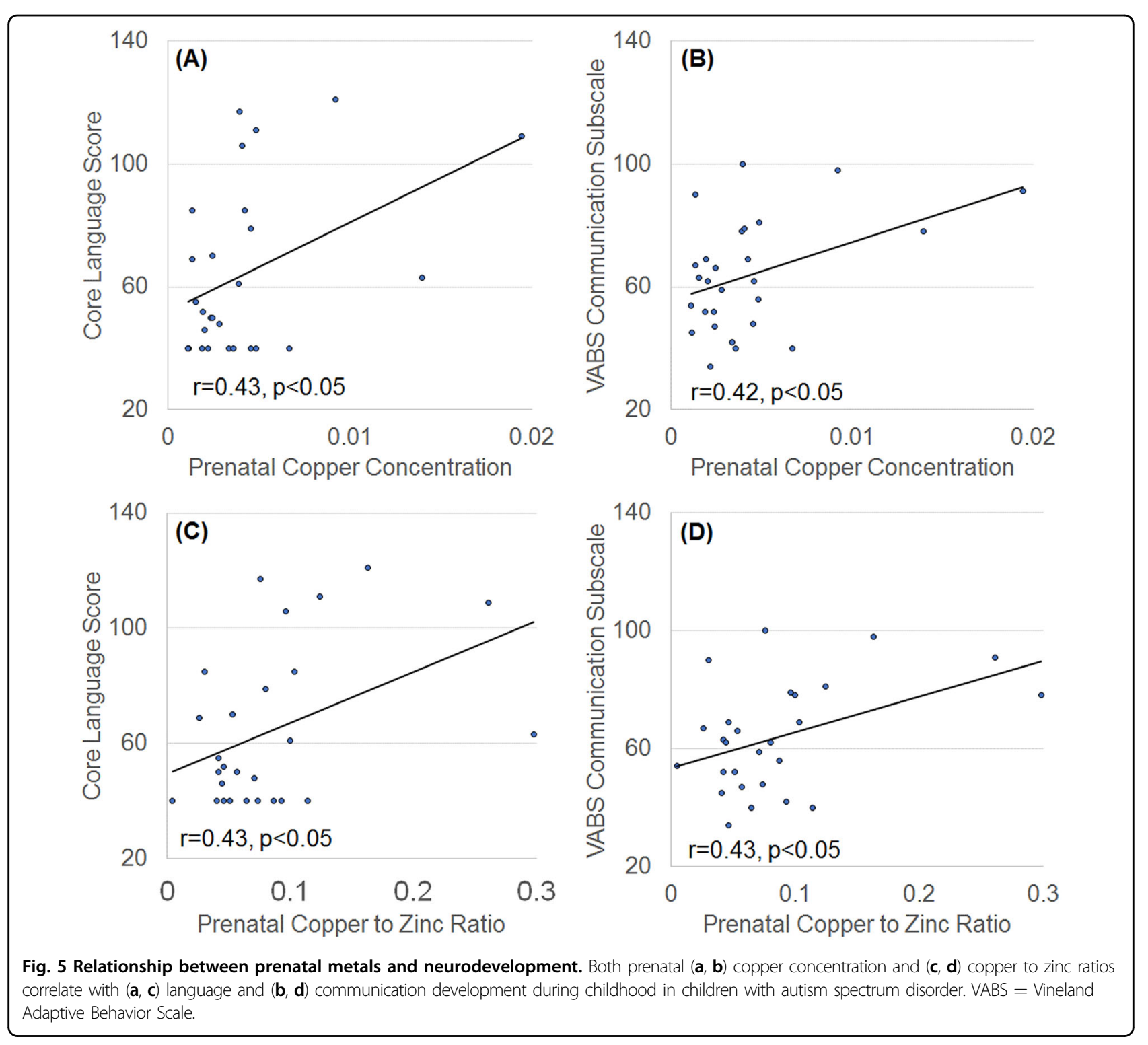


mechanism by which these metals influence neurodevelopment is not well understood in humans. As both nutritional and toxic metal exposure are known to disrupt bioenergetics and bioenergetics is disrupted in ASD, it was hypothesized that prenatal and postnatal exposure to metals was associated with long-term physiological changes in mitochondrial and glycolytic metabolism. One major strength of this study is that the exact prenatal and postnatal exposure to metals were measured by examining deciduous teeth on an individual participant basis.

We examined the prenatal and early postnatal concentrations of $\mathrm{Pb}$ (lead), $\mathrm{Ni}$ (nickel), $\mathrm{Cr}$ (chromium), $\mathrm{Mg}$ (magnesium), $\mathrm{Mn}$ (manganese), $\mathrm{Cu}$ (copper), $\mathrm{Zn}$ (zinc), $\mathrm{Sr}$ (strontium), Sn (tin), and $\mathrm{Ba}$ (barium) by analyzing deciduous teeth. First, concentrations of metals in children with ASD were compared with TD control children. Most notable was significantly lower concentrations of $\mathrm{Cu}$ in individuals with ASD as compared with control children in both the prenatal and early post-natal periods. Second, the relationship between metals and four measures of mitochondrial respiration were examined in children with ASD. Both prenatal concentrations of Mn and $\mathrm{Zn}$ were found to be significantly related to all measures of mitochondrial respiration, but only for children with a history of NDR.

The relationship between metals and glycolytic metabolism was also investigated. Both prenatal and early postnatal Mn concentration was found to be related to glycolytic rate for those children with a history of NDR. For children with a history of NDR, prenatal $\mathrm{Pb}$ was also found to be associated with glycolytic rate while for children without a history of NDR postnatal Sn was associated with glycolytic rate. Lastly, the relationship between metals and neurodevelopment and behavior were investigated. Prenatal $\mathrm{Cu}$ and the $\mathrm{Cu} / \mathrm{Zn}$ ratio, which were both abnormal prenatally in those with ASD, were found to be related to language and communication. The Pearson $r$ correlation coefficients used to describe and confirm these relationships suggest that there is a medium effect size to these relationships. These results are discussed in separate sections below.

\section{The importance of copper and zinc metabolism}

Zn deficiency has been associated with ASD both prenatally and postnatally. Two animal models of ASD involved abnormalities in $\mathrm{Zn}$ metabolism and demonstrate abnormalities in brain development. The prenatal Zn-deficient mouse model of ASD demonstrates enlargement of the thalamus ${ }^{43}$ and striatum ${ }^{44}$, and an absence of lateralization of dopamine receptor 1 in the striatum ${ }^{44}$. Male $\mathrm{Zn}$ transporter 3 null mice demonstrate ASD-type behaviors and increased cortical volume and neurite density ${ }^{45}$. Using deciduous teeth, a previous twin study demonstrated that prenatal $\mathrm{Zn}$ deficiency was related to $\mathrm{ASD}^{11}$. Several studies have demonstrated that children with ASD have lower Zn blood concentrations ${ }^{46-49}$ and have linked postnatal $\mathrm{Zn}$ concentrations during childhood to ASD severity. Indeed, one study found that hair $\mathrm{Zn}$ concentrations are inversely related to ASD symptom severity $^{50}$ and another study found that $\mathrm{Zn}$ blood levels were positively correlated with neurodevelopment ${ }^{47}$.

Perhaps more compelling, but preliminary, are studies on the potential therapeutic use of $\mathrm{Zn}$ to treat ASD behaviors. Zn supplementation improved ASD symptom severity and serum concentrations of metallothionein-1 and $\mathrm{Cu}$ in an uncontrolled cohort of children with $\mathrm{ASD}^{51}$. Despite only one patient study, there are several studies on the use of $\mathrm{Zn}$ therapeutically in animal models of ASD. Prenatal $\mathrm{Zn}$ treatment protects the prenatal valproic acid $^{52}$ and lipopolysaccharide ${ }^{53}$ exposure mouse models of ASD. Zn supplementation also prevents repetitive and anxiety behaviors in the Phelan-McDermid Syndrome mouse model of $\mathrm{ASD}^{54}$.

In this study we found that prenatal $\mathrm{Zn}$ concentrations modulates mitochondrial function in participants with a history of NDR. Recent research has identified several Zn containing low-molecular mass complexes in mitochon$\mathrm{dria}^{55}$. The major role of $\mathrm{Zn}$ with respect to mitochondrial function is also related to its function as an antioxidant through several mechanisms: the Zn-thiolate moiety of metallothioneins scavenge hydroxyl radicals, $\mathrm{Zn}$ competes with other potentially toxic metals to prevent their detrimental biological effects and, most prominently, $\mathrm{Zn}$ is a cofactor for superoxide dismutase (SOD), along with $\mathrm{Cu}$, to protect the cell from oxygen radicals ${ }^{56}$. Alternatively, high $\mathrm{Zn}$ concentrations can inhibit mitochondrial citric acid cycle and ETC enzymes ${ }^{56}$.

Abnormalities in $\mathrm{Zn}$ and $\mathrm{Cu}$ metabolism are associated with ASD with the $\mathrm{Zn}$-to- $\mathrm{Cu}$ ratio being suggested as a biomarker of $\mathrm{ASD}^{57}$. However, unlike many post-natal studies which suggest that $\mathrm{Cu}$ is elevated and the $\mathrm{Zn}$-to$\mathrm{Cu}$ ratio is low in children with $\mathrm{ASD}^{57}$, this study found that prenatal $\mathrm{Cu}$ was low and $\mathrm{Cu}$-to- $\mathrm{Zn}$ ratio was low (corresponding to a high $\mathrm{Zn}$-to- $\mathrm{Cu}$ ratio). A recent twin study examining deciduous teeth suggested that disrupted dynamics of prenatal $\mathrm{Zn}-\mathrm{Cu}$ cycles could differentiate ASD and control cases with a $90 \%$ accuracy ${ }^{12}$. Beyond the effect of $\mathrm{Cu}$ associated with $\mathrm{Zn}$ for SOD function, $\mathrm{Cu}$ is essential for the mitochondrial ETC complex IV, cytochrome oxidase ${ }^{58}$. In fact, cells contain specific machinery for transporting $\mathrm{Cu}$ into the intermembrane space of the mitochondria ${ }^{58}$. Alternatively, excess $\mathrm{Cu}$ can result in toxicity, especially in the brain ${ }^{59}$. Given the low levels of $\mathrm{Cu}$ found in the ASD participants in this study, toxicity is likely not an issue. In fact, low $\mathrm{Cu}$ concentrations may have limited the ability of the mitochondria to produce ATP, resulting in a background of mitochondrial dysfunction in the children studied. In addition, low $\mathrm{Cu}$ relative to $\mathrm{Zn}$ concentration most likely resulted in 
significant disruption of the function of important $\mathrm{Cu}-\mathrm{Zn}$ enzymes.

\section{Manganese, an important nutrient metal with potentially toxic effects}

Studies have suggested that Mn exposure is associated with neurocognitive dysfunction and psychosis ${ }^{14}$. Studies have associated higher Mn blood concentration during childhood with more severe ASD symptoms ${ }^{60}$, lower cognitive function ${ }^{50}$, and inflammatory markers ${ }^{61}$. However, using deciduous teeth, Mn concentrations have been shown to be lower in children with the ASD during the prenatal and early postnatal periods in a twin study with higher Mn concentrations correlating with less severe ASD symptoms ${ }^{11}$. Mn is essential for mitochondrial function due to its role in the mitochondrial specific SOD enzyme $^{62}$, but neurons and astrocyte mitochondria are particularly sensitive to excess $\mathrm{Mn}$ which can lead to mitochondrial dysfunction through neuroinflammation and impaired mitochondrial repair ${ }^{63}$. The data from this study suggest that a higher Mn concentration is associated with reduced mitochondrial respiration and glycolysis in ASD participants with a history of NDR. Unlike the previous study using deciduous teeth, our participants with ASD did not appear to be Mn deficient. Given the narrow therapeutic window of nutritional $\mathrm{Mn}$, the baseline $\mathrm{Mn}$ level of the participants may be an important factor associated with the modulatory effect of $\mathrm{Mn}$ on ASD severity and physiology.

\section{Lead and Tin, two metals associated with changes in glucose metabolism}

Sources of $\mathrm{Pb}$ exposure are ubiquitous in the environment, from old mine tailings, the legacy of leaded gasoline, paint deposits to soils and air pollution. Regardless of the source of $\mathrm{Pb}$, a previous twin study suggests that environmental $\mathrm{Pb}$ deposited in deciduous teeth during fetal life is higher in individuals with $\mathrm{ASD}^{11}$, and the current study suggests that it can result in long-term changes that decrease glucose metabolism in individuals with ASD and NDR. This is consistent with recent studies demonstrating that $\mathrm{Pb}$ exposure promotes diabetes ${ }^{64}$ and abnormal brain glucose metabolism ${ }^{65}$ in rodents, and may be related to the development of gestational diabetes in humans ${ }^{66}$. This study also found that early life Sn exposure was related to long-term changes in glucose metabolism in children with ASD without a history of NDR. Higher Sn concentrations have been associated with diabetes in adults ${ }^{67}$.

\section{Mitochondria dysfunction and neurodevelopmental regression}

Several studies suggest that NDR is associated with mitochondrial dysfunction ${ }^{24}$, especially in children with
$\mathrm{ASD}^{16,23}$. For example, Shoffner et al. ${ }^{23}$ showed that the majority of the children with ASD who were diagnosed with mitochondrial disease had a sudden rapid NDR, resulting in the development of ASD symptoms following an inflammatory trigger and fever. In addition, a metaanalysis of children with ASD also diagnosed with mitochondrial disease demonstrates a significantly higher rate of $\mathrm{NDR}^{16}$. We have developed a model of mitochondrial dysfunction in lymphoblastoid cell lines derived from patients with ASD where the mitochondria are more sensitive to reactive oxygen species as manifest by a significant loss of Reserve Capacity as reactive oxygen species is increased in vitro ${ }^{20,68-74}$. This subset of lymphoblastoid cell lines also respond differently to environmental agents associated with ASD including trichloroacetaldehyde hydrate $^{75}$ and the microbiome associated short-chain fattyacids propionate $\mathrm{e}^{76}$ and butyrate ${ }^{77}$. Thus, it is likely that many children with ASD and NDR have underlying mitochondrial dysfunction which makes their mitochondria sensitive to both supportive (nutritional) and detrimental (toxic) environmental influences.

\section{Limitations}

There are several limitations to this study, primarily the small sample size and the retrospective nature of the developmental history including the history of NDR. The small sample size limits the power of the statistical analyses. Thus, we cannot rule out relationships that are not found to be statistically significant. Power analysis suggests linear regression model with two ASD groups (NDR vs. no NDR) assuming a medium effect size, an alpha of 0.01 and a power of $80 \%$ requires at least 191 measurements. Our history questionnaire and follow-up clarification questions obtain a very detailed account of the NDR including developmental level prior to the NDR, the skills lost and the acuity of the event. Further research will be needed to identify the significance of these physiological changes on long-term disease.

The relationship between metal and neurodevelopment and behavior were investigated. $\mathrm{Cu}$ and the $\mathrm{Cu} / \mathrm{Zn}$ ratio was found to be related to language and communication. These metals were found to be abnormal prenatally in those with ASD but were not related to changes in mitochondrial respiration. Metals associated with changes in mitochondrial respiration were not strongly associated with behavior in this study but previous studies have documented a connection between mitochondrial function and neurodevelopment and behavior in children with $\mathrm{ASD}^{16,29}$ suggesting that the mitochondrial could be the mediator between any effect of some metals on neurodevelopment and behavior in children with ASD. The number of subjects in this study is too small to test such a hypothesis, but larger studies may be able to answer this question in the future. 


\section{Conclusion}

Although preliminary, this study demonstrates that earlylife exposure to nutritional and toxic metals can alter longterm bioenergetics, possibly resulting in disease or increasing vulnerability to disease triggered by later life events. This study found that exposure to metals during gestation was associated with variation in bioenergetic metabolism during childhood in children with ASD and NDR. The sources of the exposure have not been examined in this study. The prenatal concentrations of metals measured in deciduous teeth that were found to be significantly related to ASD, long-term change in mitochondrial respiration and/or language development (i.e., $\mathrm{Zn}, \mathrm{Cu}, \mathrm{Mn}$ ) are mostly derived from the maternal diet, suggesting that monitoring of such metals during the prenatal period may be important to ensuring optimal mitochondrial function and neurodevelopment in the fetus.

Aside from dietary sources, air pollution, which has been linked to ASD, contain many metals including naturally sourced crustal elements: $\mathrm{Ca}, \mathrm{Mg}, \mathrm{Na}, \mathrm{K}, \mathrm{Al}, \mathrm{Si}, \mathrm{Fe}, \mathrm{Ti}, \mathrm{Mn}$ and anthropogenically sourced metals: $\mathrm{V}, \mathrm{Cr}, \mathrm{Ni}, \mathrm{Zn}, \mathrm{Cd}$, $\mathrm{Pb}, \mathrm{Cu}^{78,79}$. Abnormalities in mitochondrial metabolism can be long-lasting and are implicated in a wide variety of diseases, which are suspected to have an environmental component, including psychiatric, neurodevelopmental, and neurodegenerative disorders, as well as systemic inflammation, cardiac disease, cancer, and diabetes. This study suggests that it is important to monitor the effects of sources of metal exposure on the growing fetus in order to optimize health and development.

\section{Acknowledgements}

This research was supported by the Arkansas Biosciences Institute (Little Rock, AR, USA), The Jonty Foundation (St Paul, MN), The Autism Research Institute (San Diego, CA), the Gupta Family Foundation (Atherton, CA), The Jane Bostford Johnson Foundation (New York, NY) and the Jager Family Foundation (Chicago, IL). The authors would also like to thank John Slattery for his help with coordination and Stephen Kahler for his help with patient recruitment.

\section{Author details \\ ${ }^{1}$ Barrow Neurological Institute at Phoenix Children's Hospital, Phoenix, AZ, USA ${ }^{2}$ University of Arizona College of Medicine - Phoenix, Phoenix, AZ, USA. ${ }^{3}$ North Carolina State University, Raleigh, NC, USA. ${ }^{4}$ Arkansas Children's Research Institute, Little Rock, AR, USA. ${ }^{5}$ Department of Pediatrics, University of Arkansas for Medical Sciences, Little Rock, AR, USA. ${ }^{6}$ Department of Family and Community Medicine, University of Texas Health Science Center, San Antonio, TX, USA. 'Department of Environmental Medicine and Public Health, Icahn School of Medicine at Mount Sinai, New York, NY, USA}

\section{Author contributions}

R.E.F., J.C., S.R., R.F.P. and M.A. designed the study concept. S.R., S.C.B. and S.M. performed biological samples analysis. L.D. and M.T. performed coordination and participant assessments. R.E.F., J.C., S.R., S.J.J., R.F.P. and M.A. performed data interpretation. R.E.F., J.C., S.R. and S.C.B. performed data analysis. R.E.F. and J.C. drafted the paper. All authors reviewed the paper and provided critical commentary and approved the final version of the paper.

\section{Conflict of interest}

The authors declare that they have no conflict of interest.

\section{Publisher's note}

Springer Nature remains neutral with regard to jurisdictional claims in published maps and institutional affiliations.

Supplementary Information accompanies this paper at (https://doi.org/ 10.1038/s41398-020-00905-3).

Received: 22 May 2020 Revised: 11 June 2020 Accepted: 16 June 2020 Published online: 07 July 2020

\section{References}

1. Rossignol, D. A. \& Frye, R. E. A review of research trends in physiological abnormalities in autism spectrum disorders: immune dysregulation, inflammation, oxidative stress, mitochondrial dysfunction and environmental toxicant exposures. Mol. Psychiatry 17, 389-401 (2012).

2. Zablotsky, B., Black, L. I., Maenner, M. J., Schieve, L. A. \& Blumberg, S. J. Estimated prevalence of autism and other developmental disabilities following questionnaire changes in the 2014 national health interview survey. Natl. Health Stat. Rep. 1-20 (2015).

3. Schaefer, G. B., Mendelsohn, N. J., Professional, P. \& Guidelines, C. Clinical genetics evaluation in identifying the etiology of autism spectrum disorders: 2013 guideline revisions. Genet Med. 15, 399-407 (2013).

4. Hallmayer, J. et al. Genetic heritability and shared environmental factors among twin pairs with autism. Arch. Gen. Psychiatry 68, 1095-1102 (2011).

5. Sandin, S. et al. The familial risk of autism. JAMA 311, 1770-1777 (2014).

6. Rossignol, D. A., Genuis, S. J. \& Frye, R. E. Environmental toxicants and autism spectrum disorders: a systematic review. Transl. Psychiatry 4, e360 (2014).

7. Kalkbrenner, A. E. et al. Particulate matter exposure, prenatal and postnatal windows of susceptibility, and autism spectrum disorders. Epidemiology 26, 30-42 (2015).

8. Volk, H. E., Lurmann, F., Penfold, B., Hertz-Picciotto, I. \& McConnell, R. Trafficrelated air pollution, particulate matter, and autism. JAMA Psychiatry 70, 71-77 (2013).

9. Flores-Pajot, M. C., Ofner, M., Do, M. T., Lavigne, E. \& Villeneuve, P. J. Childhood autism spectrum disorders and exposure to nitrogen dioxide, and particulate matter air pollution: a review and meta-analysis. Environ. Res. 151, 763-776 (2016).

10. Frye, R. E., Slattery, J. C. \& Quadros, E. V. Folate metabolism abnormalities in autism: potential biomarkers. Biomark. Med. 11, 687-699 (2017).

11. Arora, M. et al. Fetal and postnatal metal dysregulation in autism. Nat. Commun. 8, 15493 (2017).

12. Curtin, P. et al. Dynamical features in fetal and postnatal zinc-copper metabolic cycles predict the emergence of autism spectrum disorder. Sci. Adv. 4, eaat1293 (2018).

13. EPA. Basic information about lead air pollution. www.epa.gov, https://www. epa.gov/lead-air-pollution/basic-information-about-lead-air-pollution\#health (2018).

14. Modabbernia, A., Arora, M. \& Reichenberg, A. Environmental exposure to metals, neurodevelopment, and psychosis. Curr. Opin. Pediatr. 28, 243-249 (2016).

15. Arora, M. et al. Determining prenatal, early childhood and cumulative longterm lead exposure using micro-spatial deciduous dentine levels. PLOS ONE $\mathbf{9}$, e97805 (2014).

16. Rossignol, D. A. \& Frye, R. E. Mitochondrial dysfunction in autism spectrum disorders: a systematic review and meta-analysis. Mol. Psychiatry 17, 290-314 (2012).

17. Frye, R. E. Biomarkers of abnormal energy metabolism in children with autism spectrum disorder. N. A J. Med Sci. 5, 141-147 (2012).

18. Giulivi, C. et al. Mitochondrial dysfunction in autism. JAMA 304, 2389-2396 (2010).

19. Napoli, E., Wong, S., Hertz-Picciotto, I. \& Giulivi, C. Deficits in bioenergetics and impaired immune response in granulocytes from children with autism. Pediatrics 133, e1405-e1410 (2014).

20. Palmieri, L. et al. Altered calcium homeostasis in autism-spectrum disorders: evidence from biochemical and genetic studies of the mitochondrial aspartate/glutamate carrier AGC1. Mol. Psychiatry 15, 38-52 (2010).

21. Rose, S. et al. Clinical and molecular characteristics of mitochondrial dysfunction in autism spectrum disorder. Mol. Diagn. Ther. 22, 571-593 (2018). 
22. Bennuri, S. C., Rose, S. \& Frye, R. E. Mitochondrial dysfunction is inducible in lymphoblastoid cell lines from children with autism and may involve the TORC1 pathway. Front. Psychiatry 10, 269 (2019).

23. Shoffner, J. et al. Fever plus mitochondrial disease could be risk factors for autistic regression. J. Child Neurol. 25, 429-434 (2010).

24. Edmonds, J. L. et al. The otolaryngological manifestations of mitochondrial disease and the risk of neurodegeneration with infection. Arch. Otolaryngol. Head. Neck Surg. 128, 355-362 (2002).

25. Frye, R. E. et al. Folinic acid improves verbal communication in children with autism and language impairment: a randomized double-blind placebo-controlled trial. Mol. Psychiatry 23, 247-256 (2018).

26. Delhey, L. M. et al. The effect of mitochondrial supplements on mitochondrial activity in children with autism spectrum disorder. J. Clin. Med. 6, 18 (2017).

27. Frye, R. E. et al. Blocking and binding folate receptor alpha autoantibodies identify novel autism spectrum disorder subgroups. Front. Neurosci. 10, 80 (2016).

28. Frye, R. E. et al. Thyroid dysfunction in children with autism spectrum disorder is associated with folate receptor alpha autoimmune disorder. J. Neuroendocrinol. 29, 1-12 (2017)

29. Delhey, L. et al. Bioenergetic variation is related to autism symptomatology. Metab. Brain Dis. 32, 2021-2031 (2017).

30. Arora, M. et al. Spatial distribution of lead in human primary teeth as a biomarker of pre- and neonatal lead exposure. Sci. Total Environ. 371, 55-62 (2006).

31. Arora, M., Hare, D., Austin, C., Smith, D. R. \& Doble, P. Spatial distribution of manganese in enamel and coronal dentine of human primary teeth. Sci. Total Environ. 409, 1315-1319 (2011).

32. Arora, $M$. et al. Determining fetal manganese exposure from mantle dentine of deciduous teeth. Environ. Sci. Technol. 46, 5118-5125 (2012).

33. Andra, S. S., Austin, C., Wright, R. O. \& Arora, M. Reconstructing pre-natal and early childhood exposure to multi-class organic chemicals using teeth: towards a retrospective temporal exposome. Environ. Int. 83, 137-145 (2015).

34. Arora, M. \& Austin, C. Teeth as a biomarker of past chemical exposure. Curr. Opin. Pediatr. 25, 261-267 (2013)

35. Austin, C. et al. Barium distributions in teeth reveal early-life dietary transitions in primates. Nature 498, 216-219 (2013)

36. Gunier, R. B. et al. Determinants of manganese in prenatal dentin of shed teeth from CHAMACOS children living in an agricultural community. Environ. Sci. Technol. 47, 11249-11257 (2013).

37. Arora, M. \& Hare, D. Tooth lead levels as an estimate of lead body burden in rats following pre- and neonatal exposure. RSC Advances 5, 67308-67314 (2015).

38. Perez, J., Hill, B. G., Benavides, G. A., Dranka, B. P. \& Darley-Usmar, V. M. Role of cellular bioenergetics in smooth muscle cell proliferation induced by plateletderived growth factor. Biochem. J. 428, 255-267 (2010).

39. Hill, B. G., Higdon, A. N., Dranka, B. P. \& Darley-Usmar, V. M. Regulation of vascular smooth muscle cell bioenergetic function by protein glutathiolation. Biochim. Biophys. Acta 1797, 285-295 (2010).

40. Dranka, B. P. et al. Assessing bioenergetic function in response to oxidative stress by metabolic profiling. Free Radic. Biol. Med. 51, 1621-1635 (2011).

41. Benjamini, Y. \& Hochberg, Y. Controlling the false discovery rate: a practical and powerful approach to multiple hypothesis testing. J. R. Stat. Soc. B 57, 289-300 (1995).

42. Cohen, J. Statistical Power Analysis for the Behavioral Sciences. (Lawrence Erlbaum Associates, Publishers, Hillsdale, NJ, 1988).

43. Schoen, M. et al. Shank3 transgenic and prenatal zinc-deficient autism mouse models show convergent and individual alterations of brain structures in MRI. Front. Neural Circuits 13, 6 (2019).

44. Grabrucker, S. et al. Brain lateralization in mice is associated with zinc signaling and altered in prenatal zinc deficient mice that display features of autism spectrum disorder. Front. Mol. Neurosci. 10, 450 (2017).

45. Yoo, M. H., Kim, T. Y., Yoon, Y. H. \& Koh, J. Y. Autism phenotypes in ZnT3 null mice: Involvement of zinc dyshomeostasis, MMP-9 activation and BDNF upregulation. Sci. Rep. 6, 28548 (2016).

46. Wu, L. L., Mao, S. S., Lin, X., Yang, R. W. \& Zhu, Z. W. Evaluation of whole blood trace element levels in chinese children with autism spectrum disorder. Biol. Trace Elem. Res. 191, 269-275 (2019).

47. Guo, M., et al. Vitamin and mineral status of children with autism spectrum disorder in Hainan Province of China: associations with symptoms. Nutr. Neurosci, 1-8 (2018).
48. Qin, Y. Y. et al. A comparison of blood metal levels in autism spectrum disorder and unaffected children in Shenzhen of China and factors involved in bioaccumulation of metals. Environ. Sci. Pollut. Res. Int. 25, 17950-17956 (2018).

49. Craciun, E. C. et al. Evaluation of whole blood zinc and copper levels in children with autism spectrum disorder. Metab. Brain Dis. 31, 887-890 (2016).

50. Fiore, M. et al. Metal and essential element levels in hair and association with autism severity. J. Trace Elem. Med. Biol. 57, 126409 (2020).

51. Meguid, N. A. et al. The role of zinc supplementation on the metallothionein system in children with autism spectrum disorder. Acta Neurol. Belg. 119, 577-583 (2019)

52. Cezar, L. C. et al. Zinc as a therapy in a rat model of autism prenatally induced by valproic acid. Prog. Neuropsychopharmacol. Biol. Psychiatry 84, 173-180 (2018).

53. Kirsten, T. B., Queiroz-Hazarbassanov, N., Bernardi, M. M. \& Felicio, L. F. Prenatal zinc prevents communication impairments and BDNF disturbance in a rat model of autism induced by prenatal lipopolysaccharide exposure. Life Sci. 130, 12-17 (2015)

54. Fourie, C. et al. Dietary zinc supplementation prevents autism related behaviors and striatal synaptic dysfunction in Shank3 Exon 13-16 mutant mice Front. Cell Neurosci. 12, 374 (2018).

55. Lindahl, P. A. \& Moore, M. J. Labile low-molecular-mass metal complexes in mitochondria: trials and tribulations of a burgeoning field. Biochemistry $\mathbf{5 5}$, 4140-4153 (2016).

56. Lee, S. R. Critical role of zinc as either an antioxidant or a prooxidant in cellular systems. Oxid. Med. Cell Longev. 2018, 9156285 (2018).

57. Faber, S., Zinn, G. M., Kern, J. C. 2nd \& Kingston, H. M. The plasma zinc/serum copper ratio as a biomarker in children with autism spectrum disorders. Biomarkers 14, 171-180 (2009).

58. Baker, Z. N., Cobine, P. A. \& Leary, S. C. The mitochondrion: a central architect of copper homeostasis. Metallomics 9, 1501-1512 (2017).

59. Borchard, S. et al. The exceptional sensitivity of brain mitochondria to copper Toxicol. Vitr. 51, 11-22 (2018)

60. Rahbar, M. H. et al. Interaction between manganese and GSTP1 in relation to autism spectrum disorder while controlling for exposure to mixture of lead, mercury, arsenic, and cadmium. Res. Autism Spectr. Disord. 55, 50-63 (2018).

61. Skalny, A. V. et al. Trace element levels are associated with neuroinflammatory markers in children with autistic spectrum disorder. J. Trace Elem. Med. Biol. 50, 622-628 (2018)

62. Smith, M. R., Fernandes, J., Go, Y. M. \& Jones, D. P. Redox dynamics of manganese as a mitochondrial life-death switch. Biochem. Biophys. Res. Commun. 482, 388-398 (2017).

63. Sarkar, $\mathrm{S}$. et al. Manganese exposure induces neuroinflammation by impairing mitochondrial dynamics in astrocytes. Neurotoxicology 64, 204-218 (2018).

64. Tyrrell, J. B., Hafida, S., Stemmer, P., Adhami, A. \& Leff, T. Lead (Pb) exposure promotes diabetes in obese rodents. J. Trace Elem. Med. Biol. 39, 221-226 (2017).

65. Yun, S., Wu, Y., Niu, R., Feng, C. \& Wang, J. Effects of lead exposure on brain glucose metabolism and insulin signaling pathway in the hippocampus of rats. Toxicol. Lett. 310, 23-30 (2019).

66. Soomro, M. H. et al. Exposure to heavy metals during pregnancy related to gestational diabetes mellitus in diabetes-free mothers. Sci. Total Environ. 656, 870-876 (2019).

67. Liu, B., Sun, Y., Lehmler, H. J. \& Bao, W. Association between urinary tin concentration and diabetes in nationally representative sample of US adults. J. Diabetes 10, 977-983 (2018).

68. Rose, S. et al. Oxidative stress induces mitochondrial dysfunction in a subset of autism lymphoblastoid cell lines in a well-matched case control cohort. PLoS ONE 9, e85436 (2014)

69. Rose, S. et al. Oxidative stress induces mitochondrial dysfunction in a subset of autistic lymphoblastoid cell lines. Transl. Psychiatry 4, e377 (2014).

70. Frye, R. E. Novel cytochrome B gene mutations causing mitochondrial disease in autism. J. Ped. Neurol. 10, 35-40 (2012).

71. Frye, R. E. \& Naviaux, R. K. Autistic disorder with complex IV overactivity: a new mitochondrial syndrome. J. Pediatr. Neurol. 9, 427-434 (2011).

72. Frye, R. E., Melnyk, S. \& Macfabe, D. F. Unique acyl-carnitine profiles are potential biomarkers for acquired mitochondrial disease in autism spectrum disorder. Transl. Psychiatry 3, e220 (2013).

73. Frye, R. E. et al. Mitochondrial dysfunction may explain symptom variation in Phelan-McDermid syndrome. Sci. Rep. 6, 19544 (2016). 
74. Rose, S. et al. Mitochondrial dysfunction in the gastrointestinal mucosa of children with autism: a blinded case-control study. PLOS ONE 12, e0186377 (2017).

75. Frye, R. E. et al. Oxidative stress challenge uncovers trichloroacetaldehyde hydrate-induced mitoplasticity in autistic and control lymphoblastoid cell lines. Sci. Rep. 7, 4478 (2017).

76. Frye, R. E. et al. Modulation of mitochondrial function by the microbiome metabolite propionic acid in autism and control cell lines. Transl. Psychiatry 6 e927 (2016)
77. Rose, S. et al. Butyrate enhances mitochondrial function during oxidative stress in cell lines from boys with autism. Transl. Psychiatry 8, 42 (2018).

78. Sambandam, B. D. T., Islam, V. I. \& Prakhya, B. M. Characterization of coal fly ash nanoparticles and their induced in vitro cellular toxicity and oxidative DNA damage in different cell lines. Indian J. Exp. Biol. 53, 585-593 (2015).

79. Saikia, B. K., Saikia, J., Rabha, S., Silva, L. F. O. \& Finkelman, R. Ambient nanoparticles/nanominerals and hazardous elements from coal combustion activity: implications on energy challenges and health hazards. Geosci. Front. 9, 863-875 (2018). 